\title{
$X$-ray absorption spectroscopy for lead speciation of dispersed mine waste
}

Barry Neil Noller ${ }^{1}$, Jiajia Zheng ${ }^{1}$, Trang Huynh ${ }^{1}$, Jack Ng${ }^{2}$, Violet Diacomanolis ${ }^{2}$, Raijeli Taga ${ }^{1}$, Hugh Harris ${ }^{3}$

${ }^{1}$ The University Of Queensland, St Lucia, Australia, ${ }^{2}$ Queensland Alliance for Environmental Health Sciences, The University of Queensland, Coopers Plains, Australia, ${ }^{3}$ Department of Chemistry, The University of Adelaide, Adelaide, Australia E-mail: b.noller@uq.edu.au

The Lead Pathway Air Study (2007-2012) was a large study [1] on the human exposure to lead undertaken at Mount Isa, Queensland, Australia. Mount Isa Mines is located west of the city and has mined and processed lead-zinc and copper ores since the 1930s. The Australian health risk assessment framework [2] was followed by using bioavailability and bioaccessibility tests of lead availability together with supporting measurements using sophisticated analytical techniques. Human exposure to lead is from ingestion of $<250 \mu \mathrm{m}$ sized particles via the digestive system and inhalation of $<10 \mu \mathrm{m}$ sized particles into lungs; absorption via skin is insignificant. Extensive sampling and analysis of the mine site and city houses provided physico-chemical characteristics using synchrotron-based X-ray absorption spectroscopy (XAS) using Xray absorption near-edge spectroscopy (XANES) for estimating lead speciation/composition, high resolution lead isotope measurements and other techniques (XRD, SEM and particle size analysis) to describe multiple sources. Extensive application of lead LIII edge XANES was undertaken by recording spectra of over 300 samples at the Australian National Beamline Facility, located at the Photon Factory (KEK), Tsukuba, Japan. XANES spectra were analysed using a series of steps, as part of a well-defined statistical procedure, to determine the composition of the lead minerals or lead compounds from mineral processing present, including principal component analysis ('PCA') followed by target transformation to select the set of model compounds for linear combination fitting. Bioavailability testing on 10 composites using rats was correlated with bioaccessibility on $<250 \mu \mathrm{m}$ fractions using PBET (physiologically-based extraction test) simulating the human digestive tract. Lead solubilised in the stomach is transferred with food nutrients to the intestine where absorption occurs. Average $\mathrm{pH}$ with fast, semi-fed and full-fed states of the stomach and near neutral intestine $\mathrm{pH}$ provided an intermediate $\mathrm{pH}$ for $\mathrm{PBET}$ testing. Inhalation is $<5 \%$ of total exposure for people living in Mount Isa, while ingestion is >95\%. PM10 air particulates were not the major source of human lead exposure via inhalation in Mount Isa city for this sampling. Ingestion of $<250 \mu m$ diameter dust is from ground deposition; lead isotope ratios showed lead originated from mining Urquhart Shale and city area outcrops. The XANES analysis of PM10 air particulates exiting from the Mount Isa lead smelter stack contained negligible lead sulfide whereas near surface samples of dust and fallout from the lead smelter/ sinter plant area collected at the surface or at 2-3m above ground usually contained lead sulfide. Lead sulfide in fallout, PM10, carpet dust samples in the city indicated that some of the material collected originated from mining and/or processing activities. However, large proportions of lead-goethite in these samples made it impossible to rule out dusts from other sources, such as garden and exposed soils in the city, haul roads and tailings dams. While lead isotope ratios can show origin of lead regardless of chemical or mineral form, XANES analysis gives the chemical form. XANES analysis may show differences even when lead isotope ratios are shown to be the same.

[1] Noller et al. (2017). Lead Pathways Study - Air. Health Risk Assessment of Contaminants to Mount Isa City. 7 February 2017 Mount Isa Mines Limited, Mount Isa. pp 1-414 plus appendices.

http://www.mountisamines.com.au/EN/sustainability/Pages/LEADPATHWAYSSTUDYPORTAL.aspx

[2] enHealth (2012) Environmental Health Risk Assessment. Dept of Health and Aged Care and eNHealth Council: Canberra, Australia.

Keywords: lead, speciation, mining 